hep-th/0107263

SCIPP-01/21

hep-th/0107263

SCIPP-01/21 


\title{
String Theory, Large Dimensions and Supersymmetry*
}

\author{
Michael Dine ${ }^{a}$

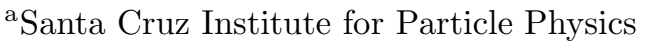 \\ University of California (Santa Cruz) \\ Santa Cruz CA 95064
}

[Invited talk presented at 30 Years of Supersymmetry, Minneapolis, October 2000]

With our current level of understanding, the problem of making string theory predictions is not one of "solving" the theory, but rather of trying to determine whether there are any generic expectations. Within this context, we discuss what it would mean to predict low energy supersymmetry, and consider questions like: what is the form of low energy $\mathrm{CP}$ violation, is unification a string prediction, and others.

\section{SUPERSYMMETRY AND STRING THEORY}

The study of supersymmetry had its beginnings in string theory, and for much of their history the two subjects have been strongly linked. The past five years have witnessed great advances in our understanding of string theory. Almost all of these have been closely tied to supersymmetry. These include:

- Dualities among string theories: Essentially all of the evidence for various dualities is based on relations which hold because of supersymmetry.

- Non-perturbative formulations of the theory in various limits: Matrix models [1] and AdS/CFT duality [2], are based on general statements, but again virtually all of the evidence provided in support of these formulations can be understood in terms of supersymmetry. It is not clear that the Matrix model proposal makes sense without supersymmetry.

- Many other developments, such as the discovery of string theories without gravity, rely heavily on supersymmetry.

Yet at the same time, we don't know whether "low energy supersymmetry" is an outcome of string theory. Indeed, while it is often claimed that low energy supersymmetry is one of the few predictions of string theory, we are far from being able to make such a statement. This is clear from Dimopoulos's talk at this meeting. Large or warped dimensions have been widely explored over the last two years as an alternative solution of the hierarchy problem[3 5]. Most scenarios of this kind do not invoke low energy supersymmetry in any conventional sense. Yet the large dimension story itself fits naturally into string theory (indeed it is hard to make sense of it without string theory).

Over the next decade, low energy supersymmetry will be confirmed or ruled out. If not observed at the LHC, our ideas about supersymmetry and the hierarchy are simply wrong. Large dimensions as a solution to the hierarchy problem are perhaps harder to rule out. We do not really have

detailed models which permit a precise phenomenology, as we do for supersymmetry. Those who contemplate two large dimensions, for example, are already confronted with a fundamental scale of order $50 \mathrm{TeV}$. Even with more dimensions, the scales are uncomfortably large from the perspective of hierarchy. So, much as for technicolor, if we accept this type of explanation, it is hard to make a precise statement of our expectations; we will simply have to await an experimental discovery (as for technicolor, we can give some qualitative experimental expectations: dense sets of states, in particular, giving rise to surprising processes at high energies). 
It would be important to be able to say: supersymmetry is a prediction (or not!) of string theory, within the next few years. It would be desirable to make a similar statement about large dimensions. We might hope to arrive at this point by "solving" string theory, and discovering that the solution has the standard model, low energy supersymmetry, unification, etc. But this is unlikely to happen soon. A more realistic hope is that we might make generic predictions, such as low energy supersymmetry, unification, some statements about $\mathrm{CP}$ or flavor. Even this is a tall order, given our current level of understanding.

In this talk, I will outline some ways in which we might be able to make a decisive statement about supersymmetry in string theory. I will argue that there is some evidence that string theory prefers supersymmetry. The evidence, currently, is tenuous, but there are ways in which we might attempt to make a firm statement. It is less clear how we might make an analogous statement about large dimensions (if we fail to make a case for supersymmetry). Short of providing some sort of solution to the theory, we would need to provide some compelling argument why such states are preferred. It seems more likely that the large dimension scenario will remain a scenario; if the large dimension story is correct, it will not be predicted, but rather (hopefully) discovered.

While I will raise a number of questions, I will provide answers only in a few cases, and even these will be, at best, conjectures. But I hope to suggest that many questions involved in relating string theory to nature are not totally out of reach. Among the questions which I will try to formulate:

- What is String Theory? This is a question we understand, in part, for theories with a large amount of supersymmetry, but for theories with little or no supersymmetry, this is far less clear.

- Some good string ground states: It is often said that the problem of determining the ground state of string theory is a dynamical one. But states with enough supersymmetry are almost certainly good ground states.
- Approximate Moduli Spaces and $\mathrm{N}=1 \mathrm{Su}$ persymmetry: If we are to formulate the question: does string theory predict low energy supersymmetry? in a generic way, we must first decide what it would mean to make such a prediction.

- Is Anything the Matter with $\mathrm{N}=0$ Supersymmetry? Having characterized the distinction between theories with low energy supersymmetry and those without, we argue that there is some evidence that theories without supersymmetry generically suffer from diseases.

- Is Small $\sin (2 \beta)$ a Supersymmetry Prediction? I will argue that ideas about supersymmetry breaking suggest that the asymmetry in $B$ decays should either be very small, or quite close to the standard model value [Recent results from the $B$ factories have ruled out the small asymmetry hypothesis.]

- Some Anthropic Issues: Given that string theory has many ground states which are drastically different from what we observe, it seems quite possible that anthropic considerations will play some role in determining the ground state in which we find ourselves.

- Coupling Unification: One of the problems with our improved understanding of string theory and its strongly coupled limits, is that it is no longer clear that coupling unification is a robust prediction.

- The Brane World in String Theory: The brane world picture is an exciting possibility, which could manifest itself in experiments at colliders. Theoretically, it poses many challenges, some of which are reviewed here. In the case of anomaly mediation, we argue that the brane world ideas are not robust.

- The role of holography: Much of the language I will use assumes a conventional effective field theory description at low en- 
ergies. As Banks has stressed, more radical proposals for the role of supersymmetry in string theory might well yield different answers to many of these questions [6]. He suggests, based on considerations of string theory in De Sitter space, that the number of degrees of freedom might be a parameter, which in turn determines the cosmological constant and the degree of supersymmetry breaking. He also argues that supersymmetry may be crucial, at least in some asymptotic sense, to attaining a consistent picture. I will not explore this intriguing possibility here (except for some later comments in the context of vacuum selection and cosmology), but just acknowledge that, given our poor understanding of the question of the cosmological constant, such radical reformulations of our thinking may well be necessary, and may lead to surprises.

\section{What is String Theory?}

Susskind[7] has proposed a provocative definition of string theory: it is that theory which lives on the moduli space of supergravity theories with a sufficiently large number of supersymmetries that we can give a non-perturbative definition (in some region of the moduli space). With our current level of understanding, this means that the number of supersymmetries must be, say, 16 or 32 , so that we can give Matrix Model or ADS/CFT definitions. This view is provocative precisely because it excludes the world we observe. I would argue that we can do much better than this.

\subsection{Good String Ground States}

But it is first worthwhile to note that string states in 4-10 dimensions with eight or more supersymmetries almost certainly exist, i.e. they are perfectly good ground states of string theory. This is simply because it is not possible to write potentials for the moduli consistent with supersymmetry. So the problem of understanding why we live in four dimensions, or why in four $\mathrm{d}$ we don't see $N>1$ is not simply a problem of deciding what is the lowest energy state, or some other well-posed dynamical question. We will engage in some speculations later.

\subsection{A Response to Susskind's Challenge}

We have at least good circumstantial evidence that in string theory, states with $N=1$ supersymmetry in four dimensions exist. More precisely, there exist approximate moduli spaces with $N=1$ supersymmetry. This evidence is based on considerations of dualities. It is much in the spirit of Witten's original discussion of duality [8].

What is meant by an approximate moduli space? Consider, e.g., some compactification of the weakly coupled heterotic string in which gluino condensation gives rise to a superpotential for the dilaton,

$W=c e^{-S / b_{o}}$.

Here $S=\frac{V}{\ell_{g}^{6} g_{s}^{2}}$, where $V$ is the volume of the compactified space, $\ell_{s}$ is the string length scale, and $g_{s}$ is the string coupling constant. In this case, there is a potential for $S$ which goes to zero as $S \rightarrow \infty$. Supersymmetry is restored in the limit as $S \rightarrow \infty$, and in particular, there is one light gravitino in this limit.

We also understand a great deal about the strongly coupled limit. The $E_{8} \times E_{8}$ heterotic string goes over to an eleven theory with two boundaries[9], separated by a distance $R_{11}$. The characteristic scale of this theory is the eleven dimensional Planck scale, $\ell_{11}$. The basic relation between the two pictures is:

$g_{s}^{2}=\frac{R_{11}^{3}}{\ell_{11}^{3}} \quad \ell_{s}^{2}=\frac{\ell_{11}^{3}}{R_{11}}$.

In the strongly-coupled picture, we can compactify six of the remaining dimensions on, say, a Calabi-Yau space characterized by a radius $R$. The moduli now are $R_{11}$ and $R$, or $g_{s}$ and $R$. It is conventional to write these as $S$ and $T$. At weak coupling,

$S=g^{-2} \frac{V}{\ell_{s}^{6}} \quad T=\frac{R^{2}}{\ell_{s}^{2}}$.

The weak coupling description is valid when

$g_{s}^{-2} \gg 1 \quad \frac{R}{\ell_{s}} \gg 1$. 
or

$\frac{S}{T^{3}} \gg 1 \quad T \gg 1$.

At strong coupling:

$T=V^{1 / 3} \frac{R_{11}}{\ell_{11}^{3}} \quad S=\frac{V}{\ell_{11}^{6}}$.

This description should be valid when

$R_{11} \gg \ell_{11} \quad V \gg \ell_{11}^{6}$,

or $\frac{T^{3}}{S} \gg 1 \quad S \gg 1$.

It is important that the regions of validity of weak and strong coupling do not overlap. However, in the effective low energy theory, one can calculate, in both regimes, certain holomorphic quantities (the superpotential and the gauge coupling function):

$$
\begin{array}{r}
f(S, T)=S+a T+\mathcal{O}\left(e^{-T}, e^{-S}\right) \\
W(S, T)=e^{-\frac{(S+a T)}{b}}+\ldots
\end{array}
$$

One can pass from weak to strong coupling keeping $S, T$ large. So these quantities should agree in these two regimes, and they do[10!! These checks are non-trivial and arguably at least as impressive as the agreement in cases with exact moduli spaces and more supersymmetry.

\section{What is Distinctive About Low Energy Supersymmetry?}

Even without Susskind's challenge, we might have asked: in what sense might string theory predict low energy supersymmetry. After all, what we "want" is to argue that nature is approximately supersymmetric. But in what sense would this be a generic outcome of string theory? What distinguishes a vacuum with approximate supersymmetry from one with no supersymmetry? Typically one speaks of theories in which "supersymmetry is unbroken at tree level." But it is unlikely that the regime of string theory which describes our world is weakly coupled, so it is unclear why "tree level" statements should have any relevance. The existence of a hierarchy does suggest, however, that we might sit at a point in an approximate moduli space. The approximate moduli spaces which admit low energy supersymmetry are distinguished by the fact that, in some limit, they become exactly supersymmetric with only four supersymmetries. This contrasts with states which become supersymmetric, if at all, only in limits with infinite numbers of supersymmetries (infinite numbers of gravitinos, for example, in some large radius limit). This discussion suggests what it would mean to show that string theory predicts low energy supersymmetry: one would want to argue that the ground state which describes the world we observe sits on one of these approximate moduli spaces.

We have at least given evidence here that such approximate moduli spaces exist. The facts suggest that if string theory describes nature, the vacuum which describes our world sits on such an approximate moduli space. How this might come about is the subject of another talk. Instead, we will try to argue that approximate moduli spaces with less supersymmetry may not make sense.

\section{States with Less Supersymmetry}

At the classical level, we know of many approximate moduli spaces in string theory where supersymmetry is restored, if at all, only in limits where there are an infinite number of gravitinos. Examples without supersymmetry include the ten-dimensional non-supersymmetric theories and toroidal compactifications of these (no supersymmetry restoration anywhere in the moduli space) and compactifications (e.g. [11]) where supersymmetry is only restored as one takes $V \rightarrow$ $\infty$.

There is some evidence that many of these theories are badly behaved:

- Most of these models have tachyons in some region of the moduli space. Thinking naively, this means that the energy (cosmological constant) is unbounded below (even if the tachyon potential has a minimum, the cosmological constant, at this point, will be of order $-\frac{1}{g^{2}}$, and thus goes to $-\infty$ as the coupling tends to zero).

- Many of these vacua are unstable, and undergo catastrophic decay 12]. These decays 
are similar to the decay of the Kaluza-Klein vacuum discussed long ago by Witten 13, in which a rip appears in spacetime, which grows at the speed of light.

- Banks has argued, from considerations of black holes and holography, that states without supersymmetry at least in some asymptotic sense, may not be consistent 6].

These observations suggest that these theories might not make sense. The problems seem generic. But with our current state of knowledge, they are not decisive. For example, we could imagine that stabilization occurs in a regime far from regions with tachyons, and that while the state is unstable, the lifetime is very long. More generally,

in a theory of gravity, as Susskind [7] and Banks 14 have stressed, it is not so easy to decide when different states are part of the same theory.

\subsection{Speculations on Other Possible Prob- lems for $\mathrm{N}=\mathbf{0}$ : Non-Perturbative Anomalies?}

We know that non-perturbative anomalies can render field theories inconsistent. It is natural to ask whether there might be such anomalies in string theories, which might render some apparently sensible states meaningless. Such a possibility, indeed, was one of the motivations for Witten's work which lead to the explosion of interest in duality 8].

However, early on, Witten 15 proved that there are no global anomalies in closed string theory, at least in the field theory limit under rather weak conditions. On the other hand, it is known that non-perturbative anomalies can lead to inconsistencies in open string theories; these problems are in some sense "dual" to violations of modular invariance in closed string theories 16.

Here we ask: are there additional consistency conditions in closed string theories? Witten's result means that we need to look at states which are far from any smooth limit. Below we briefly describe a search for anomalies in discrete symmetries.

\subsection{Are All Discrete Symmetries Gauge Symmetries?}

It is widely believed that discrete symmetries in string theories are gauged, so anomalies would signal an inconsistency. Examples include:

- Discrete symmetries of toroidal compactifications: these are remnants of higher dimensional gauge symmetries.

- $E_{8} \leftrightarrow E_{8}$ symmetry of the heterotic string: This is a subgroup of a continuous group, unbroken on a subspace of moduli space.

- T-duality of the heterotic string is, in many instances, a subgroup of a continuous gauge symmetry 17.

- CP: In many compactifications, one can see that CP is a combination of a higher dimensional Lorentz transformation and an ordinary gauge transformation 18 .

In other cases, it is less clear whether discrete symmetries are gauge symmetries. For example, some discrete symmetries of asymmetric orbifold theories, and $S$-dualities are not easily recognized as gauge symmetries. A general strategy to address this question might be the following: construct cosmic string solutions (and analogs in higher dimensions), and ask what happens if one moves particles (or more generally suitable p-branes) around them. If they pick up suitable $Z_{N}$ phases (in the case of $Z_{N}$ discrete symmetries), then the symmetries are gauged.

One construction of such cosmic strings is due to Bagger, Callan and Harvey 19. Their construction generalizes trivially to any string vacuum described by a conformal field theory with discrete symmetry $Z_{N}, N=2,3,4,6$. The idea is to compactify the $x_{2}, x_{3}$ directions on a very large torus with $Z_{N}$ symmetry, modding out by the product of the internal $Z_{N}$ and the $Z_{N}$ of the torus. The fixed points of the group action are cosmic strings. The internal $Z_{N}$ still acts nontrivially on the states of the theory. It is easy to see that particles with $Z_{N}$ charge indeed pick up a $Z_{N}$ phase as one moves about these fixed points 20. The main difficulty with this construction is the fact that these strings have a very large 
tension and correspondingly a large deficit angle. As a result, it is not totally obvious that these cosmic strings can be thought of as topological objects sitting in the original vacuum. Still, this construction is highly suggestive. An alternative construction, suggested by Banks, is under study.

\subsection{Searches for Anomalies}

The simplest way to search for anomalies in such symmetries is to examine instanton effects in the low energy effective field theory. One asks whether the instanton determinant violates the discrete symmetry. One must be careful, however, because it is often possible to cancel anomalies by a Green-Schwarz mechanism, in which one assigns a transformation law under the discrete symmetry to a modulus. Such a search was carried out in the past 21] examining symmetric orbifolds with supersymmetry. A broader search is currently in progress, including symmetric orbifolds without supersymmetry and asymmetric orbifolds, both with and without supersymmetry[20]. Currently, we have several examples of anomalies in both supersymmetric and nonsupersymmetric, asymmetric orbifolds. It seems quite possible that these indicate the existence of new non-perturbative consistency conditions in string theory. If so, it would be important to understand the stringy statement (analogous to modular invariance) of the difficulty. It is a bit disappointing that this test doesn't seem to distinguish in an obvious way between supersymmetric and non-supersymmetric theories. energy

\section{What does Supersymmetry Have to Say about $\sin (2 \beta)$}

Since this talk was presented, there have been important experimental developments. In the original talk, I argued that there were two generic predictions for $\mathrm{CP}$ violation in the $B$ system in supersymmetric theories: $\mathcal{O}\left(10^{-2}\right)$ or the standard model result (give or take about 20\%). The reasoning was quite simple 22]:

- If the $A$ parameter of order one, one needs $\mathrm{CP}$ violating phases of order $10^{-2}$ to understand $d_{n}$. In models with supersymmetry broken at an intermediate scale, one expects that the $A$ parameter is large, in general, so a natural explanation of the smallness of $d_{n}$ would be that $\mathrm{CP}$-violating phases are small. In theories of lower scale breaking, particularly gauge mediation, it is quite common for the $A$ parameter to be very small, so it is not difficult to understand $d_{n}$.

- CP must be spontaneously broken in string theory. Small spontaneous breaking - approximate $\mathrm{CP}$ - can occur naturally in such theories (simple models of this phenomenon are presented in 221). This is consistent with the high scale breaking picture in the previous item. Nir has dubbed this situation "approximate CP."

- In theories in which supersymmetry is broken at an intermediate scale (supersymmetry breaking at low energies suppressed by $\frac{1}{M_{p}}$ ), one doesn't expect excessive degeneracy of squarks and sleptons. A review of models for understanding suppression of flavor violation in supersymmetric theories was performed in 22]. Typically degeneracies of squarks are at best of order one loop factors. As a result, the real part of $K-\bar{K}$ mixing is nearly saturated by supersymmetric contributions in such models, and small phases can (should?) be responsible for the observed value of $\epsilon$. This is the case for flavor symmetries, "dilaton dominance," and "gaugino dominance" [23], and also for "anomaly mediation" 24] and "gauginomediation" [25], which are often claimed to yield much higher degrees of degeneracy (indeed, as explained in [29], there is, generically, no degeneracy).

- If $\mathrm{CP}$ is approximate, one can worry that $\frac{\epsilon^{\prime}}{\epsilon}$ will be too small. It was argued in [22], however, that $\frac{\epsilon^{\prime}}{\epsilon}$ can be of order the observed size if some of the "chirality breaking" squark mass terms are large. This is perhaps the most troubling aspect of this proposal, however. The terms mixing $\tilde{s}$ and $\tilde{d}$ must be much larger than $m_{s} \theta_{c}$. This is a natural phenomenon in theories of flavor, 
if some of the susy-breaking fields carry flavor quantum numbers. Still, it would be surprising.

- In theories with low scale breaking (e.g. gauge mediation), $A$ terms are typically very small, and the degree of squark degeneracy is very large. So supersymmetry contributions to $\epsilon$ are small, and most of the contribution must come from the Standard Model processes. There is no problem with understanding $d_{n}$, and one expects results for $\sin (2 \beta)$ close to the predictions of the standard model.

\subsection{The Experimental Situation}

Since this talk was presented, both Babar 26 and Belle 27] have announced results with greatly improved statistics for $\sin (2 \beta)$ :

Babar : $\sin (2 \beta)=0.59 \pm 0.14$.

Belle $: \sin (2 \beta)=0.99 \pm 0.14 \pm 0.06$.

This is to be compared to the standard model expectation 28:

$\sin (2 \beta)=0.75 \pm 0.2$.

So it appears that, if supersymmetry is present, the low scale option is more promising.

\section{The Problem of Vacuum Selection}

One of the remarkable features of string theory is that, classically, $N=1$ supersymmetry emerges so easily. The earliest examples of this kind were Calabi-Yau compactifications of the Type I and heterotic string theories, but the bestiary of such states quickly grew: orbifolds, fermionic models, and more intricate possibilities more recently (e.g. F-theory). In the examples we know, there is always a moduli space of vacua, classically. Many desirable features appear, such as chirality, generations, intricate discrete symmetries, candidate hidden sectors, and the like.

There are difficulties, however, both practically and conceptually, associated with the fact that there are so many states. These states are labeled by both discrete and continuous labels. So there is a vast set of states to survey, if one simply wants to look for one with a sensible phenomenology. And if one wants to determine some dynamics which fixes among these choices, almost inevitably the minimum of any potential for the moduli must lie at strong coupling or zero coupling.

These issues are illustrated by the HoravaWitten limit of string theory, the first of the "brane world" pictures. In strong coupling, the question is what fixes $R_{11}$ and $R(S$ and $T)$. Phenomenologically, one wants, in order to understand unification and the value of the Planck scale[30],

$R_{11} \sim 10-30=\quad R^{6} \sim 60$

This is an appealing picture. But it leads to a puzzle. For large $R_{11}$, the theory is approximately five dimensional, with $N=1$ supersymmetry in five dimensions. But this is enough to forbid a potential for $R_{11}$. So one expects stabilization, if at all, for $R, R_{11} \sim 1$.

There are some models for how this might come about: Kahler stabilization and the racetrack (reviewed in [31), and discrete fluxes[?]. No picture is yet totally satisfying.

\section{Coupling Unification in String Theory}

One of the triumphs of weakly coupled heterotic string theory is that whether or not one has a conventional grand unified structure at some energy scale, unification of couplings is generic. But if the string coupling is not weak, why should the couplings be unified?

The Horava-Witten picture illustrates that things are not simple. In general, the couplings do not unify. Several moduli ( $S$ and $T$ ) with large expectation values couple to gauge fields, and the $T$ couplings are not universal.

$f_{a} \approx S+c_{a} T$

where $c_{a}$ is a numerical coefficient. There are still large classes of theories for which unification occurs. In both the Kahler stabilization and racetrack pictures, one can argue for unification. But it is fair to say that we do not currently understand in what sense coupling unification is generic. 


\section{What Physics Might Choose the True Vacuum}

We have argued that there are many good vacua of string theory with totally unacceptable properties for the description of nature: more than four dimensions, unbroken supersymmetries, etc. There are even vacua with $N=1$ supersymmetry with unacceptable gauge groups 32]. What has not been established is that there are states closely resembling the standard model.

Suppose there are? Suppose Kahler stabilization, the racetrack, or something else, produces a small number of stable vacua with approximate $N=1$ supersymmetry, vanishing cosmological constant, and with certain good properties (coupling unification, proton stability,...). Why do we find ourselves in one of these? It seems to me that if this is the picture, the answer is likely to be at least partially anthropic. One can, in fact, argue that many of the vacua with too much supersymmetry will come to a bad end: structure will not form, for example. Perhaps one can make even stronger negative statements. Inflation is probably necessary in an anthropic view, but it is unlikely to be generic 33]. So perhaps there are only a small number with anything even remotely resembling the possibility of life.

We might imagine that the universe, in its history, samples all of these states, but that only this small subset is cosmologically interesting (if we are lucky, perhaps many of the unappealing ones never grow large, for example). So there might be only a small number of states which develop size and structure, and we live in this one because it is the only place we can live. The racetrack model, for example, is suggestive of this: stabilization only occurs for these theories for some finely-tuned discrete parameters. So acceptable states might not be generic.

While I won't explore this possibility further here, I think that many of the questions about the cosmology of the unappealing vacua may be accessible now, and that this is an avenue worthy of further exploration. It is interesting to note that in the proposal of Banks mentioned earlier, vacua with more than four supersymmetries are unacceptable, because they possess infinite num- bers of states.

\section{The Brane World From the Perspective of String Theory}

Prior to 1995, there were several proposals of very large ( $\mathrm{TeV}$ scale or larger) extra dimensions[3]. But it was hard to make sense of them in string theory, since the couplings were typically gigantic. With the understanding of duality, and particularly of the role of various types of branes in string theory, it became feasible to meaningfully discuss the possibility of large or warped extra dimensions 4 , 5]. The Horava-Witten model was the first example.

¿From our discussion of the Horava-Witten theory, some of the issues facing a brane world picture are clear:

- Stabilization of the moduli - in general, one expects stabilization when the moduli are of order one. The most plausible suggestion to understand larger radii is that the large dimensions are effectively two dimensional, with supersymmetry in the bulk of spacetime. Then the potential for $R$ is a function of $\ln (R)$, so the problem is just to explain why this logarithm is 20 or so. In this picture, low energy supersymmetry, as conventionally understood, is not an outcome.

- Suppression of dangerous processes: in string theory, there are no global continuous symmetries, and the effective theory typically contains a huge array of higher dimension/derivative operators, even at tree level. Rare processes, precision electroweak physics, etc. must be understood in light of this. Proposals include symmetry breaking on distant branes, discrete symmetries, and others.

- If these problems are solved (by nature, if not by us), there is a rich array of possible new phenomena (see Dimopoulos's talk at this meeting). The fundamental scale might be accessible to colliders. 


\section{Conclusions}

While I can't claim to have offered any complete answers in this talk, I have at least tried to bring into focus some generic issues, which, if resolved, might lead to string predictions:

- What is the role of approximate moduli in string theory

- Can we rule out non-susy vacua?

- What lessons can we take from cosmology, and what predictions can we make?

- Can we phrase analogous generic questions for large dimensions, Randall-Sundrum?

Lurking in the background are many issues not addressed, especially the Cosmological Constant.

\section{Acknowledgements:}

This work supported in part by a grant from the U.S. Department of Energy. I wish to thank my collaborators on the projects discussed here, particularly Alexey Anisimov, Michael Graesser, Josh Gray, Yossi Nir and Yael Shadmi. I would also like to thank Tom Banks for many discussions of issues raised here.

\section{REFERENCES}

1. W. Taylor, "Matrix Theory: Matrix Quantum Mechanics as a Fundamental Theory," to appear in Reviews of Modern Physics, hepth/0101126

2. O. Aharony, S.S. Gubser, J. Maldacena, H. Ooguri and Y. Oz, Phys.Rept. 323 (2000) 183,hep-th/9905111

3. Some precursors of these ideas can be found in V.A. Rubakov and M.E. Shaposhnikov, "Do We Live Inside A Domain Wall?," Phys. Lett. 125B, 136 (1983); G. Dvali and M. Shifman, "Dynamical compactification as a mechanism of spontaneous supersymmetry breaking," Nucl. Phys. B504, 127 (1997), hepth/9611213; G. Dvali and M. Shifman, "Domain walls in strongly coupled theories," Phys. Lett. B396, 64 (1997); (E)B407, 452,1997, hep-th/9612128.
4. N. Arkani-Hamed, S. Dimopoulos and G. Dvali, "The Hierarchy Problem and New Dimensions at a Millimeter," Phys. Rev. Lett. B429 (1998) 263, hep-ph/9803315; . Antoniadis, N. Arkani-Hamed, S. Dimopoulos and G. Dvali, "New dimensions at a millimeter to a Fermi and superstrings at a TeV," Phys. Lett. B436, 257 (1998) hep$\mathrm{ph} / 9804398$. Some precursors of these ideas can be found in [3].

5. L. Randall and R. Sundrum, "A Large Mass Hierarchy From a Small Extra Dimension," hep-ph/9905221; L. Randall and R. Sundrum, "An Alternative to Compactification," hepth/9906064.

6. T. Banks, "Cosmological breaking of Supersymmetry, or Little Lambda Goes Back to the Future II," hep-th/0007146.

7. Leonard Susskind, private communication.

8. E. Witten, "String Theory Dynamics in Various Dimensions," Nucl. Phys. B443 (1995) 85, hep-th/9503124.

9. P. Horava and E. Witten, "ElevenDimensional Supergravity on a Manifold With Boundary," Nucl.Phys. B475 (1996) 94, hep-th/9603142; "Heterotic and Type I String Dynamics from ElevenDimensions," Nucl.Phys. B460 (1996) 506, hep-th/9510209.

10. T. Banks and M. Dine, "Couplings and Scales in Strongly Coupled Heterotic String Theory," Nucl.Phys. B479 (1996) 173, hepth/9605136.

11. R. Rohm, "Spontaneous Supersymmetry Breaking in Supersymmetric String Theories," Nucl.Phys. B237 (1984) 553.

12. M. Fabinger and P. Horava, "Casimir Effect Between World Branes in Heterotic M Theory," Nucl.Phys. B580 (2000) 243, hepth/0002073.

13. E. Witten, "Instability of the Kaluza-Klein Vacuum," Nucl.Phys. B195 (1982) 481.

14. T. Banks, "On Isolated Vacua and Background Independence," hep-th/0011255.

15. E. Witten, "Topological Tools in TenDimensional Physics," in Unified String Theories, M. Green and D. Gross editors, World Scientific (1985) Singapore. 
16. M. Berkooz, R.G. Leigh, J. Polchinski, N. Seiberg and E. Witten, "Anomalies, Dualities and Topology of $D=6, N=1$ Superstring Vacua," Nucl.Phys. B475 (1996) 115, hep-th/9605184.

17. A. Giveon, E. Rabinovici and G. Veneziano, "Duality in String Background Space," Nucl.Phys. B322 (1989) 167; M. Dine, P. Huet and N. Seiberg, "Large and Small Radius in String Theory," Nucl.Phys. B322 (1989) 301.

18. K. Choi, D.B. Kaplan and A.E. Nelson, "Is CP a Gauge Symmetry," Nucl.Phys. B391 (1993) 515, hep-ph/9205202; M. Dine, R.G. Leigh and D.A. MacIntire, "Of CP and Other Gauge Symmetries in String Theory," Phys.Rev.Lett. 69 (1992) 2030, hepth/9205011.

19. J.A. Bagger, C.G. Callan, and J.A. Harvey, "Cosmic Strings as Orbifolds," Nucl. Phys. B278 (1986) 550.

20. M. Dine, M. Graesser and J. Gray, in preparation.

21. D.A. MacIntire, "Investigation of Discrete Gauge Anomalies in String Theory," hepth/9308149.

22. M. Dine, E. Kramer, Y. Nir and Y. Shadmi, "CP Violation and the Scale of Supersymmetry Breaking," hep-ph/0101092.

23. M. Dine, A. Kagan and S. Samuel, "Naturalness in Supersymmetry, or Raising the Supersymmetry Breaking Scale," Phys.Lett. B243 (1990) 250.

24. L. Randall and R. Sundrum, "Out of This World Supersymmetry Breaking," Nucl.Phys. B557 (1999) 79, hep-th/9810155; G. Giudice, M.A. Luty, H. Murayama and R. Barbieri, "Gaugino Mass Without Singlets," JHEP 9812 (1998) 027, hep-ph/9810442

25. D.E. Kaplan, G.D. Kribs and M. Schmaltz, "Supersymmetry Breaking Through Transparent Extra Dimensions," Phys.Rev. D62 (2000) 035010, hep-ph/9911293; Z. Chacko, M.A. Luty, A.E. Nelson and E. Ponton, JHEP 0001 (2000) 003, hep-ph/9911323; M. Schmaltz and W. Skiba, "The Superpartner Spectrum of Gaugino Mediation," Phys.Rev. D62 (2000) 095004, hep-ph/0004210.
26. B. Aubert et al (BABAR Collaboration), "Observation of CP Violation in the $B^{o} \mathrm{Me}-$ son System," hep-ex/0107013.

27. K. Abe et al (for the Belle Collaboration), "Observation of Large CP Violation in the Neutral B Meson System," hep-ex/0107061.

28. See, for example, the article of Gilman et al in D.E. Groom et al, "Review of Particle Properties," Eur.Phys.J. C15 (2000) 1.

29. A. Anisimov,M. Dine and M. Graesser, SCIPP preprint in preparation.

30. E. Witten, "Strong Coupling Expansion of Calabi-Yau Compactification," Nucl.Phys. B471 (1996) 135, hep-th/9602070

31. M. Dine, "Some Reflections on Moduli, Their Stabilization and Cosmology," hepth/0001157; "Towards a Solution of the Moduli Problems of String Cosmology," Phys.Lett. B482 (2000) 213, hep-th/0002047.

32. T. Banks and M. Dine, "Quantum Moduli Spaces of N=1 String Theories," Phys.Rev. D53 (1996) 5790, hep-th/9508071.

33. T. Banks, "Remarks on M Theoretic Cosmology," hep-th/9906126. 